\title{
Resonant gravity-wave drag enhancement in linear stratified flow over mountains
}

Article

Accepted Version

Teixeira, M. A. C., Miranda, P. M. A., Argain, J. L. and Valente, M. A. (2005) Resonant gravity-wave drag enhancement in linear stratified flow over mountains. Quarterly Journal of the Royal Meteorological Society, 131 (609). pp. 1795-1814. ISSN 1477-870X doi: https://doi.org/10.1256/qj.04.154 Available at https://centaur.reading.ac.uk/29251/

It is advisable to refer to the publisher's version if you intend to cite from the work. See Guidance on citing.

To link to this article DOI: http://dx.doi.org/10.1256/qj.04.154

Publisher: Royal Meteorological Society

All outputs in CentAUR are protected by Intellectual Property Rights law, including copyright law. Copyright and IPR is retained by the creators or other copyright holders. Terms and conditions for use of this material are defined in the End User Agreement.

\section{www.reading.ac.uk/centaur}

\section{CentAUR}

Central Archive at the University of Reading 
Reading's research outputs online 


\title{
Resonant gravity wave drag enhancement in linear stratified flow over mountains
}

\author{
By M. A. C. Teixeira ${ }^{1 *}$, P. M. A. Miranda ${ }^{1}$, J. L Argain ${ }^{2}$ and M. A. Valente ${ }^{1}$ \\ ${ }^{1}$ Geophysics Centre and Department of Physics, University of Lisbon, Portugal \\ ${ }^{2}$ Department of Physics, University of Algarve, Faro, Portugal
}

(Received 1 January 2002; revised 1 January 2002)

\section{SUMMARY}

High-drag states produced in stratified flow over a $2 \mathrm{D}$ ridge and an axisymmetric mountain are investigated using a linear, hydrostatic, analytical model. A wind profile is assumed where the background velocity is constant up to a height $z_{1}$ and then decreases linearly, and the internal gravity wave solutions are calculated exactly. In flow over a $2 \mathrm{D}$ ridge, the normalised surface drag is given by a closed-form analytical expression, while in flow over an axisymmetric mountain it is given by an expression involving a simple 1D integral. The drag is found to depend on two dimensionless parameters: a dimensionless height formed with $z_{1}$, and the Richardson number in the shear layer, Ri. The drag oscillates as $z_{1}$ increases, with a period of half the hydrostatic vertical wavelength of the gravity waves. The amplitude of this modulation increases as $R i$ decreases. This behaviour is due to wave reflection at $z_{1}$. Drag maxima correspond to constructive interference of the upward propagating and downward propagating waves in the region $z<z_{1}$, while drag minima correspond to destructive interference. The reflection coefficient at the interface $z=z_{1}$ increases as $R i$ decreases. The critical level, $z_{c}$, plays no role in the drag amplification. A preliminary numerical treatment of nonlinear effects is presented, where $z_{c}$ appears to become more relevant, and flow over a $2 \mathrm{D}$ ridge qualitatively changes its character. But these effects, and their connection with linear theory, still need to be better understood.

KEYwORDS: Mountain waves, High-drag states, Linear theory

\section{INTRODUCTION}

A substantial amount of the literature devoted to the study of orographic gravity waves in the atmosphere deals with the problem of resonant flows, high-drag states and downslope windstorms. This is presumably due to the severe effects that these flows have on local weather and on gravity wave drag. Downslope windstorms may increase the value of the drag exerted by the wind on a mountain ridge by one order of magnitude relative to the leading-order, linear estimate (Bacmeister and Pierrehumbert 1988). The impact of such states on the globally integrated drag, and hence on the deceleration of the global atmospheric circulation, may be large. For the purpose of parameterising gravity wave drag in large-scale numerical models, it is therefore useful to understand what determines the onset of high-drag states. Since these states arise in flow over mountains of relatively high amplitude, the physical mechanisms responsible for them are thought to be intrinsically nonlinear. As a consequence, most of the studies addressing this problem have been numerical (Clark and Farley 1984, Peltier and Clark 1983, Clark and Peltier 1984, Bacmeister and Pierrehumbert 1988, Miranda and Valente 1997). However, our understanding of these physical mechanisms is still incomplete. Simplified models are usually helpful in providing insight into these mechanisms, despite often having unrealistically restrictive assumptions. They isolate individual physical processes, and enable a more exhaustive exploration of the parameter space.

Conceptual models have been proposed to explain the existence of highdrag states and downslope windstorms. One of them, developed by Clark and

\footnotetext{
* Corresponding author: Centro de Geofísica da Universidade de Lisboa, Edifício C8, Campo Grande, 1749-016 Lisbon, Portugal.

(c) Royal Meteorological Society, 2002.
} 
Peltier (1984) claims that the reflection of gravity waves at environmental or self-induced critical levels in strongly nonlinear flows leads to a resonance process. Clark and Peltier's model gives the heights for which a critical level produces high-drag states (with a periodicity of half the hydrostatic vertical wavelength), but does not specify the magnitude of the drag or its variation between high-drag states. Additionally, the assumption of reflection of the waves is not solidly founded. Another model, developed by Smith (1985), is based on a hydraulic analogy, and explains high-drag states as a result of the existence of the equivalent of a hydraulic jump in the flow. The model does not solve the flow equations in the whole domain and uses relatively crude assumptions. Unlike Clark and Peltier's model, Smith's model correctly predicts the periodicity of one hydrostatic wavelength for the critical level heights that produce high-drag states. It also gives an indication of the drag magnitude based on a simplified momentum budget. However, the upper boundary condition applied to Long's equation is somewhat arbitrary and the model does not predict the variation of the drag between high-drag states.

Smith's theory has been supported by the subsequent numerical simulations of Bacmeister and Pierrehumbert (1988) using environmental critical levels, which also raised some new issues. For example, these authors found that high-drag states may exist even for mountains of small dimensionless height, but their steady state takes a longer time to be attained. In this case, the phase of the reflection from the critical level is also different from that predicted by Smith (1985). There is some controversy regarding the role played by the Richardson number at the critical level in the establishment of high-drag states. Scinocca and Peltier (1991) claimed that the onset of these states is delayed or even prevented for relatively low Ri. Additionally, these authors questioned the importance of the resonance shift introduced by Smith, whereby the height of the critical levels leading to resonance increases as the dimensionless height of the mountain increases. These claims have been criticised by Durran (1992), who proved the existence of a resonance shift in nonlinear calculations using Long's equation. In these calculations, a critical level was not present, but resonance conditions were produced by considering a two layer atmosphere, with different Brunt-Väisälä frequencies in each layer. More recently, Miranda and Valente (1997) addressed the case of flow with an environmental critical level over an axisymmetric mountain, showing that in this 3D configuration, the resonant drag enhancement and resonance shift are reduced relative to the $2 \mathrm{D}$ case, and the flow is closer to linear. They also found that the periodicity of the high-drag states as a function of the height of the critical level is of one half the hydrostatic vertical wavelength, in agreement with Clark and Peltier's (1984) arguments. These results suggest that some interesting insights into these processes can be obtained using linear theory.

Indeed, explanations for downslope windstorms based on the variation of the wind and static stability with height have been sought in the framework of linear theory. Using multiple-layer models, Klemp and Lilly (1975) and Peltier and Clark (1979), explored the flow configurations that maximise the surface wind. But their studies were not focused on the surface drag. Using a related approach, Wang and Lin (1999a, 1999b), following Lindzen and Tung (1976), studied the effects of wave ducting by shear layers for a wide range of Richardson numbers. They suggested that the heights where the wind velocity has a discontinuity in its first derivative may be more important than the height of the critical level. They 
argued that this might partially explain the phenomenon that was perceived by previous authors as resonance shift. They also stressed the role of the Richardson number in the shear layer as a key parameter of the flow, with an importance much beyond that of delaying the onset of high-drag states.

Grimshaw and Smyth (1986) suggested that high-drag states may begin with linear resonance, and Wang and Lin (1999a) found that, when the Richardson number is above 0.25 , the influence of what goes on above a critical level is very limited. So, in this study a very simple linear model is proposed, which may help to elucidate how the high-drag states are initiated. It considers a wind profile that is constant up to a certain level, and above decreases linearly, reaching a critical level. Although analogous (in fact slightly more complicated) wind profiles were used in the studies of Lindzen and Tung (1976) and Wang and Lin (1999a,1999b), among others, the main focus of these authors was not on the surface drag, and analytical expressions for this quantity in resonant flows have not been derived. In this study, the surface drag is calculated, and found to be given, for flow over a ridge, by a closed analytical formula that depends on two parameters: the height of the level where the first derivative of the wind profile is discontinuous and the Richardson number in the shear layer above. The present approach is similar to that of Leutbecher (2001), where, however, the more familiar problem of an environment with discontinuities in the Brunt-Väisälä frequency was investigated.

This paper is organised as follows: section 2 presents the theoretical model, in section 3 the results from this model are compared with numerical simulations, and the flow structure is analysed. In section 4 the main conclusions are presented.

\section{LiNEAR FLOW WITH A CRITICAL LEVEL}

In this study, inviscid, non-rotating and hydrostatic flow over an isolated mountain is considered. For typical windspeeds, and provided that conditions for the occurrence of lee waves are absent, this is a valid approximation for mountains with horizontal scales considerably larger than $1 \mathrm{~km}$ and smaller than $100 \mathrm{~km}$. These are the scales that need to be parameterised in global circulation models. Additionally, the flow is assumed to be linear, which typically is acceptable for mountains much lower than $1000 \mathrm{~m}$. Since the mountains that satisfy this condition produce a relatively small fraction of the globally integrated drag, this assumption is made mainly for mathematical convenience. Nevertheless, even when this and the other assumptions are not formally satisfied, one hopes that the physical processes associated with the simplified situations where they apply carry over to more general situations.

It is also assumed that the static stability, expressed by the Brunt-Väisälä frequency of the flow, $N$, is constant. The purpose of this is to isolate the effect of the vertical wind shear on mountain waves. From the fundamental equations of fluid mechanics subject to the Boussinesq approximation and to the above assumptions, a single equation may be derived for the vertical velocity perturbation associated with the internal gravity waves generated by the mountain. Taking Fourier transforms of the flow perturbations (which may be done if they decay to zero sufficiently fast away from the mountain), the Fourier transform of the vertical velocity perturbation, $\hat{w}$, satisfies, in a stationary situation, the Taylor-Goldstein equation (Sawyer 1962):

$$
\hat{w}^{\prime \prime}+\left[\frac{N^{2} k_{12}^{2}}{\left(U k_{1}+V k_{2}\right)^{2}}-\frac{U^{\prime \prime} k_{1}+V^{\prime \prime} k_{2}}{U k_{1}+V k_{2}}\right] \hat{w}=0 .
$$


Here the primes denote differentiation with respect to $z,(U, V)$ is the background or unperturbed wind (assumed to depend only on $z),\left(k_{1}, k_{2}\right)$ is the horizontal wavenumber vector of the internal gravity waves described by (1) and $N=$ $\left[\left(g / \theta_{0}\right) d \theta_{0} / d z\right]^{1 / 2}$ is the Brunt-Väisälä frequency of the flow. In this expression, $g$ is the acceleration of gravity, $\theta_{0}$ is the background or unperturbed potential temperature (also assumed to depend only on $z$ ) and $k_{12}=\left(k_{1}^{2}+k_{2}^{2}\right)^{1 / 2}$ is the magnitude of the horizontal wavenumber.

The background flow is considered to be:

$$
\begin{aligned}
U & =\left\{\begin{array}{l}
U_{0} \text { if } z \leqslant z_{1} \\
U_{0}\left(\frac{z_{c}-z}{z_{c}-z_{1}}\right) \quad \text { if } \quad z>z_{1},
\end{array}\right. \\
V & =0
\end{aligned}
$$

where $U_{0}$ is the windspeed at the surface, $z_{1}$ is the height where the first derivative of the background velocity has a discontinuity and $z_{c}$ is the critical level (where $U=0$ ). This wind profile, which near the ground is constant and then decreases linearly, is similar to the profiles used by Lindzen and Tung (1976), Miranda and Valente (1997) and Wang and Lin (1999a, 1999b) up to the critical level. At the critical level height, or some distance above, those authors assumed the windspeed to become again constant.

The present wind profile is also approximately similar, in a qualitative sense, to the hyperbolic-tangent profiles considered in the studies of Bacmeister and Pierrehumbert (1988) and Scinocca and Peltier (1991). Both profiles possess a zone of approximately constant velocity and a zone of maximum negative curvature (which in the present case is infinite), topped by a critical level. It will be seen next that, for the range of Richardson numbers considered here, what goes on above the critical level has little influence on what goes on below, so that the difference between the present wind profile and those used in the previous studies mentioned above is not very relevant.

For the velocity profile (2)-(3), the solution to (1) in the region $0<z<z_{1}$ is:

$$
\hat{w}=\alpha\left(k_{1}, k_{2}\right) e^{i \frac{N k_{12}}{U_{0} k_{1}} z}+\beta\left(k_{1}, k_{2}\right) e^{-i \frac{N k_{12}}{U_{0} k_{1}} z},
$$

where $\alpha$ and $\beta$ are functions to be determined. The first term corresponds to a wave whose energy propagates upward and the second term to a wave whose energy propagates downward. These two terms must be considered because wave reflection may occur at $z_{1}$. In the region $z>z_{1}$, the solution to (1) is (cf. Grubišić and Smolarkiewicz 1997):

$$
\hat{w}=\gamma\left(k_{1}, k_{2}\right)\left(\frac{z_{c}-z}{z_{c}-z_{1}}\right)^{1 / 2-i \mu \operatorname{sgn}\left(k_{1}\right)},
$$

where $\gamma$ is another function to be determined, $\mu=\left(R i k_{12}^{2} / k_{1}^{2}-1 / 4\right)^{1 / 2}$, and $R i$ is the Richardson number of the flow, defined here as $R i=N^{2}\left(z_{c}-z_{1}\right)^{2} / U_{0}^{2}$. In (5) it is implicitly assumed that $R i>1 / 4$, which is the necessary condition for hydrodynamic stability of the flow (Miles 1961), and also that the wave energy propagates upward. This is consistent with previous treatments using a backward linear velocity profile (Smith 1986, Grubišić and Smolarkiewicz 1997) and is also intuitively correct for a wind that remains linear up to infinity: in the linear approximation and for $R i>1 / 4$, the critical level does not reflect any wave energy but only absorbs it. 
In most of what follows, only $R i>0.5$ will be considered. According to Grubišsic and Smolarkiewicz (1997), the attenuation of the wave amplitude as it passes through the critical level is $\exp \left[-\pi\left(R i k_{12}^{2} / k_{1}^{2}-1 / 4\right)^{1 / 2}\right]$. So, a lower bound for this attenuation when $R i=0.5$ is 0.20 . Having in mind that, in order to influence the flow below the critical level, a wave component has to cross it upward and then downward, and in the second process it undergoes a further attenuation of the same magnitude, this wave component should have 0.04 of its initial amplitude. This shows that what happens above the critical level is essentially irrelevant for this range of $R i$, and justifies the use of a two-layer model. These arguments are also consistent with the results of Wang and Lin (1999a). They note that the characteristics of their third uppermost layer (where the wind is again constant) have little impact on the reflection and transmission coefficients for $R i$ larger than $O(1)$ (see their Figs. 9 and 11).

The Taylor-Goldstein equation (1) is subject to 3 boundary conditions, which determine the three unknown functions $\alpha, \beta$ and $\gamma$. The first condition states that the wind is tangential to the ground at the surface:

$$
\hat{w}(z=0)=i U_{0} k_{1} \hat{\eta}
$$

where $\hat{\eta}$ is the Fourier transform of the terrain elevation, $\eta(x, y)$. The second boundary condition states that the normal velocity at the (perturbed) interface existing at $z \approx z_{1}$ is continuous. Since, from (2), the background wind is continuous at $z=z_{1}$, this amounts to requiring that $\hat{w}$ be continuous at $z_{1}$. The third boundary condition requires that the pressure (or its Fourier transform) be continuous at $z=z_{1}$. The Fourier transform of the pressure perturbation is given by

$$
\hat{p}=i \frac{\rho_{0}}{k_{12}^{2}}\left[\left(U^{\prime} k_{1}+V^{\prime} k_{2}\right) \hat{w}-\left(U k_{1}+V k_{2}\right) \hat{w}^{\prime}\right],
$$

where $\rho_{0}$ is a reference density (assumed constant). So, this last boundary condition can also be expressed in terms of $\hat{w}$ and $\hat{w}^{\prime}$.

When these boundary conditions are applied to (4)-(5), one obtains

$$
\begin{array}{r}
\alpha=\frac{U_{0} k_{1} \hat{\eta}\left[i N \frac{k_{12}}{k_{1}}+\frac{U_{0}}{z_{c}-z_{1}}\left(\frac{1}{2}+i \mu\right)\right] e^{-i \frac{N k_{12}}{U_{0} k_{1}} z_{1}}}{2 N \frac{k_{12}}{k_{1}} \cos \left(\frac{N k_{12}}{U_{0} k_{1}} z_{1}\right)-2 \frac{U_{0}}{z_{c}-z_{1}}\left(\frac{1}{2}+i \mu\right) \sin \left(\frac{N k_{12}}{U_{0} k_{1}} z_{1}\right)}, \\
\beta=\frac{U_{0} k_{1} \hat{\eta}\left[i N \frac{k_{12}}{k_{1}}-\frac{U_{0}}{z_{c}-z_{1}}\left(\frac{1}{2}+i \mu\right)\right] e^{i \frac{N k_{12}}{U_{0} k_{1}} z_{1}}}{2 N \frac{k_{12}}{k_{1}} \cos \left(\frac{N k_{12}}{U_{0} k_{1}} z_{1}\right)-2 \frac{U_{0}}{z_{c}-z_{1}}\left(\frac{1}{2}+i \mu\right) \sin \left(\frac{N k_{12}}{U_{0} k_{1}} z_{1}\right)}, \\
\gamma=\frac{i U_{0} \hat{\eta} N k_{12}}{N \frac{k_{12}}{k_{1}} \cos \left(\frac{N k_{12}}{U_{0} k_{1}} z_{1}\right)-\frac{U_{0}}{z_{c}-z_{1}}\left(\frac{1}{2}+i \mu\right) \sin \left(\frac{N k_{12}}{U_{0} k_{1}} z_{1}\right)} .
\end{array}
$$

This totally specifies the solution to the present problem: the other flow variables may be straightforwardly obtained from $\hat{w}$ and its vertical derivative, as will be seen later.

\section{(a) Reflection coefficient}

In order to understand the behaviour of the wave solutions, it is useful to calculate the reflection coefficient at $z_{1}$. Following Wang and Lin (1999a), this is 
defined as the modulus of the ratio of the amplitude of the downward propagating and the upward propagating wave in the lower layer. From (8)-(9), it may be shown that this is

$$
R=\left|\frac{\beta}{\alpha}\right|=\left[\frac{1-\left(1-\frac{1}{4 R i} \frac{k_{1}^{2}}{k_{12}^{2}}\right)^{1 / 2}}{1+\left(1-\frac{1}{4 R i} \frac{k_{1}^{2}}{k_{12}^{2}}\right)^{1 / 2}}\right]^{1 / 2}
$$

The reflection coefficient does not depend on the phase of the waves, as shown by the fact that (11) is independent of $z_{1}$. However, it depends on the Richardson number and on the horizontal wavenumber, more exactly on its azimuthal direction. Generally, it decreases as the Richardson number increases and as $k_{1} / k_{12}$ decreases, being zero for $R i \rightarrow \infty$ or $k_{1} / k_{12}=0$. For $R i=1 / 4$, and $k_{2} / k_{12}=0$, $R=1$.

This means that a larger fraction of the waves generated by the mountain is reflected at $z=z_{1}$ as $R i$ decreases, which is intuitively correct, since there is a progressively larger contrast in vertical wind shear between the two layers considered (in the limit $R i \rightarrow \infty$ there is no contrast at all). On the other hand, only waves with wavenumbers in the direction of the mean flow $\left(k_{2}=0\right)$ undergo the maximum amount of reflection possible. Waves perpendicular to this direction pass through the level $z=z_{1}$ unmodified (cf. Grubišić and Smolarkiewicz 1997). Therefore, in the linear regime, the dynamical significance of $R i$, which Scinocca and Peltier (1991) wondered about, is that of controlling the intensity of the wave reflection at $z_{1}$. (As remarked earlier, the equivalent to $z_{1}$ for the flow used by those authors is the location of the maximum in wind profile curvature.) This behaviour has consequences for the surface drag, as will be seen next.

\section{(b) Gravity wave drag}

For the wind profile (2)-(3), the vertical flux of horizontal wave momentum is constant up to the critical level, in accordance with Eliassen-Palm's theorem, and equal in magnitude to the surface pressure drag. It is the divergence of this flux, in the vicinity of the critical level, that must be parameterised in large-scale numerical models. However, this study is concerned instead with the total surface drag.

From (4) and (7), the Fourier transform of the pressure perturbation at the surface is given by

$$
\hat{p}(z=0)=\frac{\rho_{0} N}{k_{12}}\left[\alpha\left(k_{1}, k_{2}\right)-\beta\left(k_{1}, k_{2}\right)\right],
$$

which may be calculated using the definitions (8)-(9). The pressure drag, defined as the integral of the pressure perturbation times the terrain slope integrated over the whole domain considered, may also be expressed in spectral space as

$$
D=4 \pi^{2} i \int_{-\infty}^{+\infty} \int_{-\infty}^{+\infty} k_{1} \hat{p}^{*}(z=0) \hat{\eta} d k_{1} d k_{2},
$$

where the asterisk denotes complex conjugate. This definition only pertains to the drag along $x$, since the $y$ component of the drag is zero for the background flow and orography chosen in this study. In order to calculate the integrals in (13), it is necessary to know the form of the Fourier transform of the terrain elevation, 
$\hat{\eta}$. However, since the drag for a constant wind in the hydrostatic approximation is well-known (e.g. Smith 1980, Phillips 1984), this study is concerned primarily with departures from this value. Therefore, in what follows, $D$ is normalised by $D_{0}$, the hydrostatic linear drag for a constant wind. It turns out that the dimensionless quantity $D / D_{0}$ does not depend on the detailed shape of the orography, as long as this is exactly axisymmetric (cf. Teixeira et al. 2004). This property arises from the the fact that, in hydrostatic flow, the pressure corrections due to the vertical wind variation do not depend on the wavenumber value, but only on its azimuthal direction.

Introducing (8)-(9) into (12) and (12) into (13), and adopting the polar coordinates to calculate the integrals,

$$
k_{1}=k_{12} \cos \theta, \quad k_{2}=k_{12} \sin \theta,
$$

it may be shown that the normalised drag takes the form

$$
\frac{D}{D_{0}}=\frac{1}{\pi} \int_{0}^{2 \pi} \frac{\cos ^{2} \theta\left(1-\frac{1}{4 R i} \cos ^{2} \theta\right)^{1 / 2}}{1-\frac{1}{2} R i^{-1 / 2} \cos \theta \sin \left(\frac{2 N}{U_{0} \cos \theta} z_{1}\right)} d \theta
$$

This simple expression is obtained because the integral over the wavenumber magnitude $k_{12}$ in (13) may be separated from the integral over $\theta$, and cancels out when the drag is divided by $D_{0}$. Additionally, only the imaginary part of $\hat{p}$ contributes to (15), as is clear from (13) and having in mind that the drag is a real quantity.

Several features stand out from this formula. Firstly, the normalised drag depends on two dimensionless parameters: $R i$ and $N z_{1} / U_{0}$ (not $N z_{c} / U_{0}$ ). The drag is modulated, oscillating with $N z_{1} / U_{0}$ with a period of nearly half the hydrostatic vertical wavelength, in agreement with the theory of Clark and Peltier (1984) and the numerical simulations of Miranda and Valente (1997). The Richardson number in the shear layer determines the amplitude of this modulation, making it larger for small $R i$. This modulation is a manifestation of the fact that the lower layer in the present model acts like a resonant cavity. Waves that are generated by the mountain are partially reflected at $z=z_{1}$, then totally reflected at the surface (because the wave energy must remain in the atmosphere), then again partially reflected at $z=z_{1}$, and so on. Note that the important height in this process is $z_{1}$ and not $z_{c}$, in accordance with the arguments of Wang and Lin (1999a, 1999b). So the drag modulation can be attributed totally to the discontinuity in the shear. The irrelevance of reflections at $z_{c}$ in a linear model like the present one is, of course, not surprising, since the construction of the corresponding wave solutions automatically rules them out. In the numerator of (15), Ri also has the effect of generally decreasing the value of the drag (independently of $N z_{1} / U_{0}$ ). When $z_{1}=0$, the background flow reduces to that imposed by Grubišić and Smolarkiewicz (1997), and consequently the drag also has the form found by these authors, decreasing as $R i$ decreases. When $R i \rightarrow \infty, D / D_{0} \rightarrow 1$, as would be expected.

A more thorough analysis of the drag behaviour is left for section 3 , where these analytical results are compared with numerical simulations. 


\section{(c) 2D flow}

A comparison between the behaviour of the drag in flow over $3 \mathrm{D}$ and $2 \mathrm{D}$ mountains is quite relevant, because it has been shown that this behaviour differs markedly in strongly nonlinear circumstances (Miranda and Valente 1997).

It is straightforward to adapt the previous calculations to flow over a 2D ridge, for which all the flow variables display $2 \mathrm{D}$ symmetry and are given by $1 \mathrm{D}$ instead of $2 \mathrm{D}$ Fourier integrals. For this purpose, it is sufficient to set $k_{2}=0$ and note that, as a consequence, $k_{12}=k_{1}$. In that case, $\mu$ is redefined as $\mu=(R i-1 / 4)^{1 / 2}$ and the solutions for $\hat{w},(4)-(5)$, as well as the coefficients $\alpha, \beta$ and $\gamma,(8)-(10)$, are modified accordingly. The boundary conditions to apply at $z=0$ and $z=z_{1}$ are the same as in the 3D case.

From the modified definitions of $\alpha$ and $\beta$, the reflection coefficient at $z=z_{1}$ may be derived:

$$
R=\left|\frac{\beta}{\alpha}\right|=\left[\frac{1-\left(1-\frac{1}{4 R i}\right)^{1 / 2}}{1+\left(1-\frac{1}{4 R i}\right)^{1 / 2}}\right]^{1 / 2} .
$$

Due to the absence of wave dispersion, $R$ no longer depends on the horizontal wavenumber, but only on $R i$, and varies between $R=0$ for $R i=\infty$ and $R=1$ for $R i=1 / 4$. Hence for $R i=1 / 4$ there is total reflection at $z=z_{1}$. This result is consistent with Figs. 4 and 11 of Wang and Lin (1999a), but this comparison should be viewed with caution. For $R i=1 / 4$, the critical level is perfectly transparent to gravity waves and thus discontinuities in the derivatives of the wind profile above must have an impact on the reflection coefficient. This is not taken into account in the present model. It is also interesting to note that the reflection coefficient (16) is equal to that in Eq. (32) of Keller (1994), although the wind profile used by that author was linear in the lower layer and constant above.

The gravity wave drag must now be defined as a drag per unit width of the ridge (since the total drag would be infinite). In spectral space, the drag is given by

$$
D=2 \pi i \int_{-\infty}^{+\infty} k_{1} \hat{p}^{*}(z=0) \hat{\eta} d k_{1} .
$$

For similar reasons as in the 3D case (the Fourier transform of the correction to the pressure perturbation does not depend on $k_{1}$ ) the expressions involving the vertical variation of the wind profile may be taken outside the integral. So, when $D$ is divided by $D_{0}$ (which now corresponds to the well-known drag per unit length of uniform hydrostatic flow over a ridge) (Queney 1948, Smith 1986), the integral cancels out. The expression of the normalised drag $D / D_{0}$ is thus totally analytical and independent of the form of $\hat{\eta}$ :

$$
\frac{D}{D_{0}}=\frac{\left(1-\frac{1}{4 R i}\right)^{1 / 2}}{1-\frac{1}{2} R i^{-1 / 2} \sin \left(\frac{2 N}{U_{0}} z_{1}\right)} .
$$

This expression shows, even more clearly than (15), the influence of the resonance process on the drag modulation. The drag maxima now occur exactly for $N z_{1} / U_{0}=\pi / 4+\pi n$, where $n$ is an integer number, and the minima for $N z_{1} / U_{0}=3 \pi / 4+\pi n$. The amplitude of the modulation is controlled by the Richardson number qualitatively in the same way as in the $3 \mathrm{D}$ case, and the 
drag also becomes generally smaller as $R i$ decreases in the numerator of (18). When $z_{1}=0$, and the velocity profile is linear down to the surface, (18) reduces to Eq. (3.17) of Smith (1986). When $R i \rightarrow 1 / 4$, the denominator of (18) oscillates between 2 and 0 , making the drag maxima tend to infinity. However, because the numerator also tends to zero as $R i \rightarrow 1 / 4$, the drag minima tend to zero and the width of the maxima becomes very small. These aspects are discussed in more detail in section $3(\mathrm{a})$.

\section{COMPARISON WITH NUMERICAL RESUlTS}

In this section, the analytical model developed above for the calculation of gravity wave drag is compared with data resulting from simulations of $2 \mathrm{D}$ and 3D, nonlinear, non-hydrostatic mesoscale numerical models. Details about the 3D model, called NH3D, are provided in Miranda (1991) and Miranda and James (1992), and details about the 2D model, called FLEX, can be found in Argain (2003). The models are initially run for approximately linear and hydrostatic conditions, in order to test the drag expressions previously derived. Inviscid conditions are also considered, and the models are run until a steady state is attained.

\section{(a) Linear flow over a $2 D$ ridge}

The FLEX model is used to simulate flow over a 2D ridge. Many studies exist of this situation, but generally considering highly nonlinear conditions. A grid of $150 \times 200$ points, with a spacing of $1708 \mathrm{~m}$ in the horizontal and $86 \mathrm{~m}$ in the vertical, is employed. The drag is averaged over the last third of the integration time.

Figure 1 compares the normalised drag for the flow (2)-(3) (lines) with numerical simulation data (symbols), for $R i=2, R i=1$ and $R i=0.5$. In the numerical runs, a bell shaped ridge with height $h_{0}=10 \mathrm{~m}$ and half-width $a=16 \mathrm{~km}$ is used, such that $N h_{0} / U_{0}=0.01$ and $N a / U_{0}=16$, so the flow is very nearly linear and hydrostatic. The solid line and the squares correspond to $R i=2$, the dotted line and the diamonds to $R i=1$ and the dashed line and the circles to $R i=0.5$.

The drag oscillates periodically with $N z_{1} / \pi U_{0}$, having a dimensionless period of 1 , which when expressed in terms of $z_{1}$ is $\pi U_{0} / N$, or half the hydrostatic vertical wavelength of the gravity waves. The drag maxima are located at $N z_{1} / \pi U_{0}=$ $1 / 4+n$, where $n$ is an integer, and the drag minima at $N z_{1} / \pi U_{0}=3 / 4+n$. The locations of the drag maxima coincide precisely with those obtained for the 'linear duct modes' associated with a strong surface response of the flow, by Wang and Lin (1999b). They differ from the locations of the maxima predicted by Clark and Peltier (1984), which were expressed in terms of the critical level height as $N z_{c} / \pi U_{0}=0.5+n$. Wang and Lin (1999a) reconciled these two results by noting how $z_{c}$ and $z_{1}$ are related for the simple flow considered here: $N z_{c} / \pi U_{0}=N z_{1} / \pi U_{0}+R i^{1 / 2} / \pi$. For example, if $R i=1$ the difference between the two normalised heights is 0.32 , which is relatively close to the value necessary for them to be consistent (0.25). Admittedly, for $R i=2.25$ (which is used in e.g. Clark and Peltier 1984), the difference is too large (0.48), but that study used a hyperbolic-tangent wind profile, where the relation between $z_{1}$ and $z_{c}$ is harder to define.

It is interesting to note that, in a model applicable to an atmosphere with discontinuous $N$ (Leutbecher 2001), the shape of the resonant drag curves is 


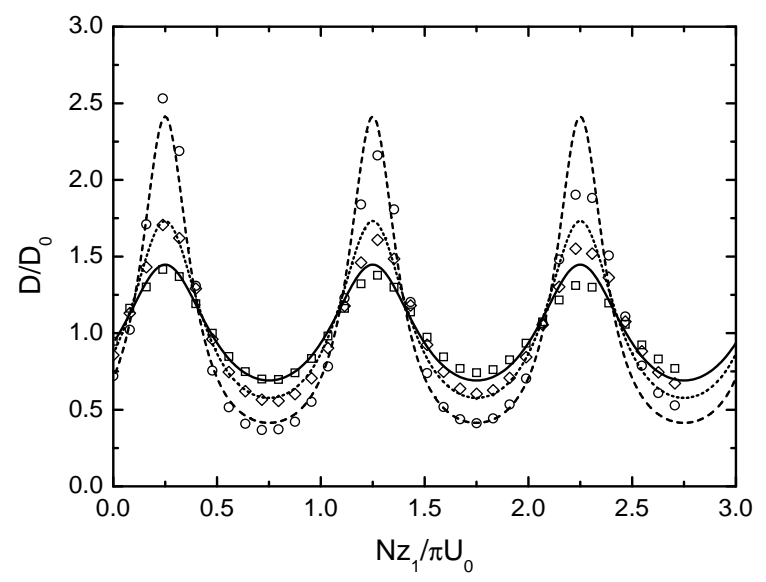

Figure 1. Normalised drag as a function of the shear layer height for flow over a 2D ridge. Lines: linear theory (18), symbols: FLEX numerical model. Solid line and squares: $R i=2$, dotted line and diamonds: $R i=1$, dashed line and circles: $R i=0.5$.

very similar to that displayed in Fig. 1, but the maxima occur instead at exact integer values of the dimensionless height of the interface separating the layers with different $N$. This similarity is due to the fact that the drag behaviour in that case also results from constructive or destructive interference of vertically reflected waves.

In Fig. 1, the amplitude of the drag modulation increases as $R i$ decreases. This is consistent with the finding by Wang and Lin (1999b) that the lowlevel response of the gravity waves (diagnosed through the velocity perturbation) strengthens as $R i$ decreases. However, Wang and Lin did not explicitly calculate the drag. Through analysis of (18), it can be shown that the maximum and minimum values of the drag (corresponding, respectively to the peaks and to the troughs of the lines in Fig. 1) are given, respectively, by

$$
\frac{D_{\max }}{D_{0}}=\left(\frac{1+\frac{1}{2} R i^{-1 / 2}}{1-\frac{1}{2} R i^{-1 / 2}}\right)^{1 / 2}, \quad \frac{D_{\min }}{D_{0}}=\left(\frac{1-\frac{1}{2} R i^{-1 / 2}}{1+\frac{1}{2} R i^{-1 / 2}}\right)^{1 / 2}=\left(\frac{D_{\max }}{D_{0}}\right)^{-1} .
$$

These values are plotted in Fig. 2(a) as a function of $R i^{-1}$. It can be seen that $D_{\max } \rightarrow+\infty$ and $D_{\min } \rightarrow 0$ as $R i \rightarrow 1 / 4$.

While the analytical drag displays a perfectly periodic behaviour in Fig. 1, the numerical results show a slightly decreasing amplitude of the modulation for the largest values of $N z_{1} / \pi U_{0}$. This is probably a spurious consequence of the fact that the momentum flux at high levels takes a long time to attain a steadystate, or is damped numerically. For this reason, the transmission of the waves in the lower layer becomes progressively more imperfect as $z_{1}$ increases. It is also possible that the wind profile above the critical level has a small influence on the surface drag for $R i=0.5$ (it was seen in section 2 that the critical level becomes progressively more transparent to waves as $R i \rightarrow 1 / 4$ ).

Because the numerator of (18) tends to zero when $R i \rightarrow 1 / 4$, the width of the drag maxima becomes progressively smaller relative to the width of the minima as $R i$ decreases (the peaks of the curve become narrower than the troughs). This effect was also verified in the study of Leutbecher (2001). One way to estimate 

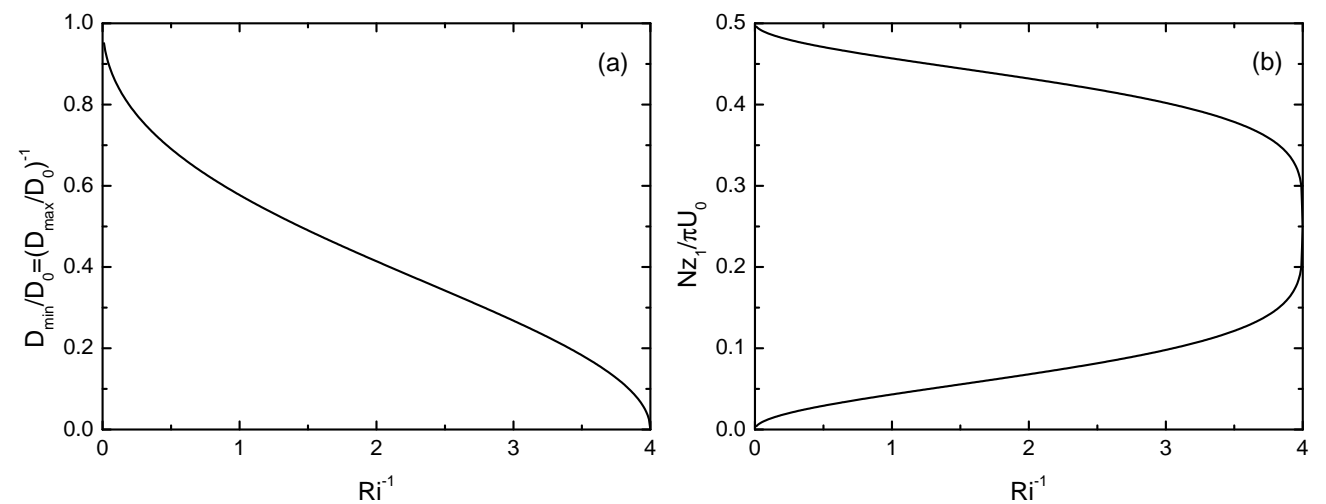

Figure 2. (a) Maximum and minimum normalised drag as a function of $R i^{-1}$ for flow over a $2 \mathrm{D}$ ridge. (b) Lower and upper limits of the first maximum in Fig. 1 (defined as the heights where the normalised drag equals 1$)$ as a function of $R i^{-1}$.

the width of the maxima is by determining the heights for which the drag takes the normalised value of 1 . These heights, which delimit consecutive maxima and minima of the drag, are found to be given by the solution to

$$
\sin \left(\frac{2 N}{U_{0}} z_{1}\right)=2 R i^{1 / 2}\left[1-\left(1-\frac{1}{4 R i}\right)^{1 / 2}\right] .
$$

As $R i \rightarrow+\infty$, the maxima tend to have the same width as the minima, since the spacing of the roots of (20) becomes uniform.

Figure 2(b) shows the solutions to (20) in the interval $[0,0.5]$, which correspond to the lateral limits of the first maximum in Fig. 1 (the solution would repeat itself periodically after $N z_{1} / \pi U_{0}=1$ ). When there is no asymmetry between peaks and troughs, the limits of the first maximum are at $N z_{1} / \pi U_{0}=0$ and $N z_{1} / \pi U_{0}=0.5$, respectively. Figure. 2(b) shows that these limits move closer together as $R i$ decreases, which signals the narrowing of the maxima. This phenomenon might provide a tentative explanation for the remark by Scinocca and Peltier (1991) that high-drag states take a long time to be attained or may even not arise when $R i$ is relatively small. Since the drag maxima become narrower as $R i$ decreases, a finer flow tuning might be required for them to be realised.

The good agreement of the analytical results and the numerical results in Fig. 1 confirms that, for hydrostatic waves of small amplitude, the two parameters that control the drag are $N z_{1} / \pi U_{0}$ and $R i$. This supports the view by Wang and Lin (1999b) that $z_{1}$ is a better indicator than $z_{c}$ for the occurrence of high-drag states. On the other hand, it supports the claim by Scinocca and Peltier (1991) that $R i$ (at the critical level) is a key governing parameter of the flow. As remarked above, Scinocca and Peltier focused on the effect of $R i$ on the time evolution of the drag. The present results suggest that a much more important role is played by $R i$ (which was hinted at in the studies of Wang and Lin 1999a,1999b): that of controlling the amplitude of the drag modulation.

\section{(b) Flow structure}

Some insight into the reasons for the observed drag behaviour may be obtained by analysing the associated velocity field. This is done here using 
the same analytical model that was developed above to calculate the drag. Vertical cross-sections displaying the normalised streamwise (along $x$ ) velocity perturbation, $u /\left(N h_{0}\right)$, and the total potential temperature, $\theta_{0}+\Theta$, are plotted next. $u$ is obtained by taking the inverse Fourier transform of $\hat{u}$ using an FFT algorithm (Press et al. 1992). $\hat{u}$ is given by

$$
\hat{u}=i \frac{\hat{w}^{\prime}}{k_{1}},
$$

which results from the conservation of mass (in 2D), and may be calculated from (4)-(5) and (8)-(10). The total potential temperature, which is the sum of the background potential temperature and the corresponding perturbation, $\Theta$, can be calculated using the buoyancy perturbation, $b=g \Theta / \theta_{0}$. The buoyancy perturbation is given by the inverse Fourier transform of

$$
\hat{b}=i \frac{N^{2} \hat{w}}{U k_{1}},
$$

which results from the heat balance equation. In Fig. $3, b$ has been amplified consistent with a dimensionless mountain height of $N h_{0} / U_{0}=0.5$ in order to facilitate the flow visualisation. Lines of constant total potential temperature (sometimes called isentropes) correspond to streamlines, since the flow is assumed to be adiabatic.

A bell-shaped ridge given by

$$
\eta=\frac{h_{0}}{1+(x / a)^{2}}
$$

is assumed to be the obstacle that generates the waves.

Figure 3(a) corresponds to a case of no resonance where $R i=\infty$, Fig. 3(b) to a case where there is maximum resonant drag enhancement $\left(N z_{1} / \pi U_{0}=2.25\right.$ and $R i=0.5$ - third peak of the dashed line in Fig. 1), and Fig. 3(c) to a case where there is maximum resonant drag reduction $\left(N z_{1} / \pi U_{0}=2.75\right.$ and $R i=0.5$ - third trough of the dashed line in Fig. 1).

In Fig. 3(a), the typical structure of linear, hydrostatic internal gravity waves can be seen, with phase lines sloping in the upwind direction, and the disturbance confined above the mountain. The flow resembles that of the classical solution of Queney (cf. Fig. 6 of Wurtele et al. 1996 and Fig. 2 of Peltier and Clark 1979). $u$ has a maximum on the downwind slope of the ridge, which corresponds to a minimum of $p$ (in fact, in a constant-wind layer, the normalised streamwise velocity and pressure have the same value but opposite signs). The isentropes display at the surface a slight asymmetry relative to the ridge, which corresponds to a normalised drag of $\approx 1$. Due to linearisation, the surface streamline does not exactly coincide with the terrain elevation profile.

Figure 3(b) shows that, in the on-resonance case, $u$ is much larger than in the no-resonance case. The $u$ dipole at the surface, with negative values upwind and positive values downwind of the mountain, is considerably stronger. Due to constructive interference of the upward and downward-propagating waves, the $u$ maxima and minima now possess two lobes, which reinforce the anti-symmetry of $u$ relative to the mountain. The isentropes clearly possess a much stronger asymmetry than in the no-resonance case, reflecting the fact that the normalised drag is $\approx 2.5$. Although showing a somewhat smaller deflection, the isentrope 

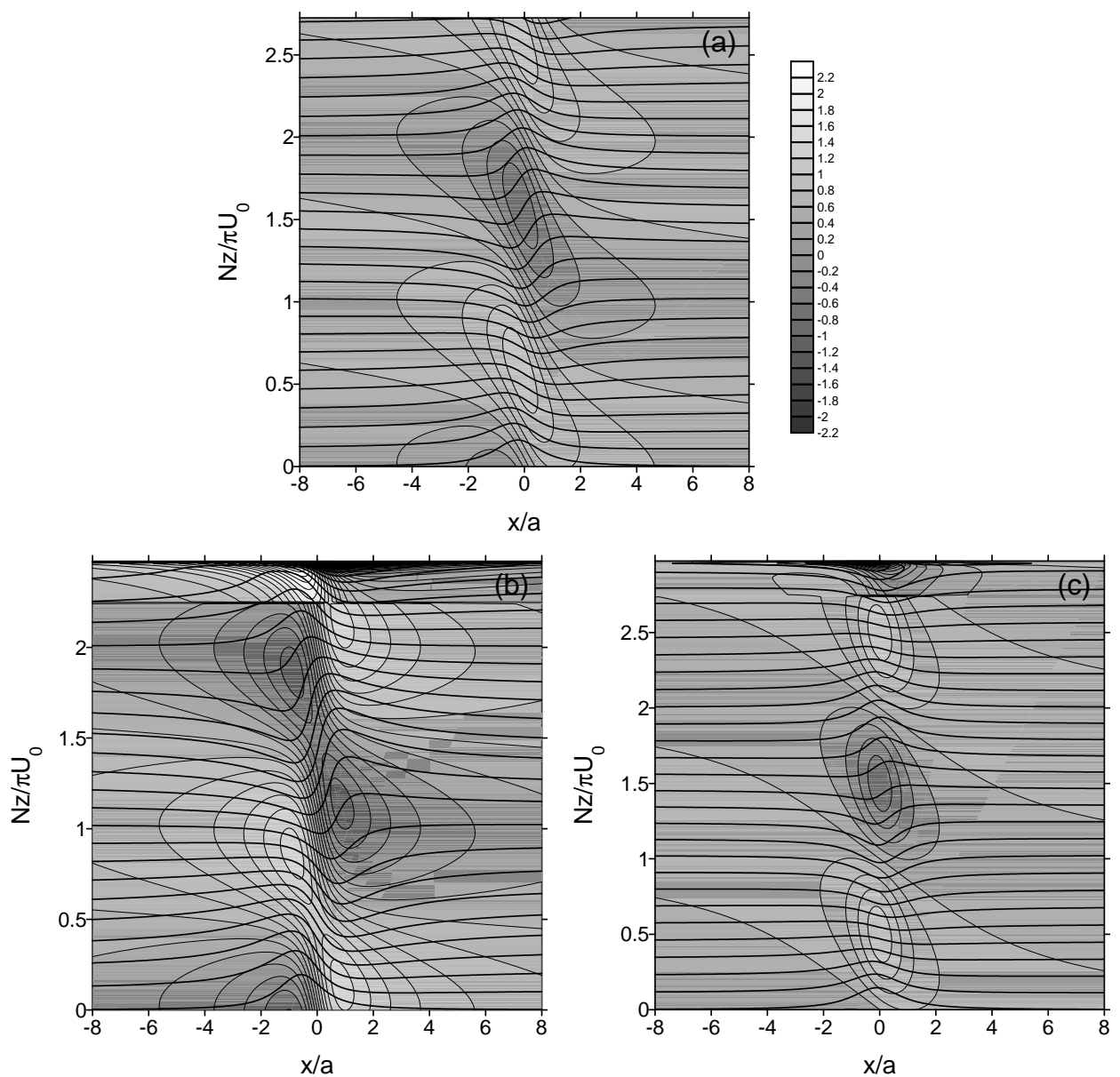

Figure 3. Vertical cross-sections of the normalised streamwise velocity perturbation, $u /\left(N h_{0}\right)$, (shaded contours) and total potential temperature - assuming $N h_{0} / U_{0}=0.5$ to facilitate visualization - (thick solid lines), from linear theory. Isentrope spacing: $1 \mathrm{~K}$. (a) $R i=\infty$, (b) $R i=0.5$ and $N z_{1} / \pi U_{0}=2.25$,

(c) $R i=0.5$ and $N z_{1} / \pi U_{0}=2.75$. In (b) and (c) the upper limit of the domain is the critical level.

configuration in Fig. 3(b) clearly resembles that associated with high-drag states in Fig. 5 of Bacmeister and Pierrehumbert (1988). The isentropes are not only visibly much closer together on the downwind side of the mountain, but they also have a larger slope throughout the flow, indicating that wave breaking is more likely than in the no-resonance case, even far from the critical level.

In Fig. 3(c) it can be seen that, in the off-resonance case, $u$ is weaker and more symmetric relative to the mountain than in the no-resonance case. This leads to a weaker pressure perturbation, that generates a dimensionless drag $\approx 0.5$. The isentropes are less deflected than either in the no-resonance or the on-resonance case. The flow is therefore farther from breaking conditions, and the asymmetry of the isentropes near the surface is very small. This is consistent with the low observed drag, and also with Fig. 6 of Bacmeister and Pierrehumbert (1988), where a low-drag state is illustrated.

Since sometimes it is not easy to distinguish whether a given flow is characterised by wave absorption or reflection at a certain level, Fig. 3 gives hints as 
to what qualitative features of the flow one should look for in the $u$ field when reflection does exist.

\section{(c) 3D flow over an axisymmetric mountain}

In this section, the NH3D model is run for flow over an axisymmetric mountain, in order to test the corresponding analytical drag expression derived before, (15). The domain of integration consists of $65 \times 65 \times 200$ gridpoints, with a horizontal spacing of $4 \mathrm{~km}$. The vertical coordinate is a pressure-based sigma coordinate, with grid spacing between $38 \mathrm{~m}$ at the surface and $90 \mathrm{~m}$ near the top of the domain. The drag is averaged over the final third of the integration time. Once again, very nearly hydrostatic and linear conditions are considered $\left(N h_{0} / U_{0}=0.01\right.$ and $\left.N a / U_{0}=16\right)$, so as to focus only on wind profile effects.

Figure 4 shows results of the NH3D model (symbols) compared with results from (15) (lines). The normalised drag oscillates with $N z_{1} / \pi U_{0}$, as in the $2 \mathrm{D}$ case, but the drag curves are now not strictly periodic: the amplitude of the drag modulation decreases as $N z_{1} / \pi U_{0}$ increases and the period is not exactly, but only approximately, of $\pi U_{0} / N$. As pointed out by Leutbecher (2001), who treated analogous 2D and 3D situations, these differences in behaviour are due to the fact that 3D gravity waves are dispersive (the vertical wavenumber depends on the azimuthal direction of the horizontal wavenumber). For this reason, the drag modulation is also generally of lower amplitude than in the $2 \mathrm{D}$ case, and the drag maxima do not tend to infinity when $R i \rightarrow 1 / 4$. This happens because the singularity in the denominator of (15) is integrable. Additionally, there is a slight asymmetry of the drag modulation curves, with a relatively slow rise to the maxima and a relatively faster fall to the minima for high $N z_{1} / \pi U_{0}$, especially at $R i=0.5$. A similar effect was observed in the results of Leutbecher (2001).

Although wave dispersion now explains a part of the decay of the amplitude of the modulation as $N z_{1} / \pi U_{0}$ increases, there is, as in the 2D case, an excessive dispersive decay in the numerical data which should be attributed to the fact that the momentum flux probably was spuriously damped at the higher levels of the domain.

The maxima of the drag in the numerical runs occur for slightly smaller values of $N z_{1} / \pi U_{0}$ than in the analytical formula. (An analogous effect, albeit in the opposite direction and of much smaller magnitude is visible in Fig. 1 for the $2 \mathrm{D}$ case). It is possible that this is due to the limited vertical resolution of the numerical model. This is consistent with the observation that this shift increases as $N z_{1} / \pi U_{0}$ increases, since the vertical resolution of the NH3D model becomes coarser (the vertical spacing of the grid levels becomes larger) as the height increases.

These results show that, in the linear regime, the main differences between resonance in flow over a $2 \mathrm{D}$ ridge and over an axisymmetric mountain are the relatively smaller magnitude of the high-drag states in the latter case and the fact that their amplitude decays as $N z_{1} / \pi U_{0}$ increases. The first aspect is noticeable in Fig. 6(a) of Miranda and Valente (1997) (for a nonlinear flow regime) while the accuracy of existing numerical simulations is insufficient to be sure about the second aspect. Figure 5 of Clark and Peltier (1984) and Fig. 2 of Bacmeister and Pierrehumbert (1988) certainly suggest (for nonlinear conditions) that the amplitude of consecutive drag maxima is constant in $2 \mathrm{D}$ flow, but more tests are needed. At this point, one important question arises: what features of linear flow are retained in more realistic, nonlinear circumstances? 


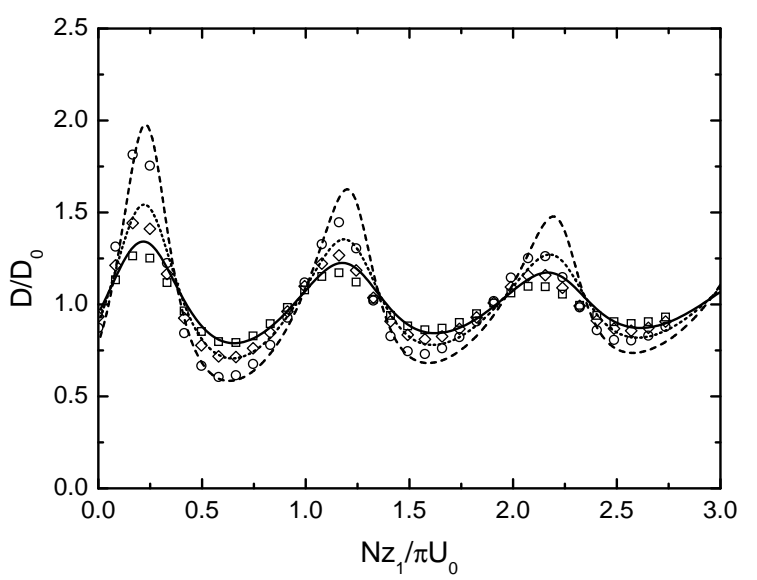

Figure 4. Normalised drag as a function of the shear layer height for flow over an axisymmetric mountain. Lines: linear theory (15), symbols: NH3D numerical model. Solid line and squares: $R i=2$, dotted line and diamonds: $R i=1$, dashed line and circles: $R i=0.5$.

\section{(d) Nonlinear effects}

The numerical simulations of Miranda and Valente (1997) showed that, in the nonlinear regime, the drag amplification produced in flow over an axisymmetric mountain is more modest than in flow over a $2 \mathrm{D}$ ridge. The drag maxima have a spacing of half rather than one hydrostatic vertical wavelength and there is also considerably less resonance shift. The flow appears to be more linear and qualitatively consistent with Clark and Peltier's (1984) resonance theory. Since the previous linear results suggest much smaller differences, it would be interesting to explore the transition between linear and nonlinear high-drag regimes. This section will not attempt more than a preliminary step in that direction.

The FLEX and NH3D numerical models were run for the same Richardson numbers as previously, but using $N h_{0} / U_{0}=0.5$. This dimensionless mountain height, while probably insufficient for wave breaking in the constant-wind layer, is sufficient for the flow to take a distinctly nonlinear character.

Figures 5(a,b) show results for flow over a ridge and Figs. 5(c,d) for flow over an axisymmetric mountain. In Figs. 5(a,c) the usual scaling for the horizontal axis, involving $z_{1}$, is used, and the vertical lines correspond to the locations of maxima predicted from the present linear model: $N z_{1} / \pi U_{0}=0.25+n$ for $2 \mathrm{D}$ flow and $N z_{1} / \pi U_{0} \approx 0.20+n$ for $3 \mathrm{D}$ flow (where $n$ is an integer). In Figs. $5(\mathrm{~b}, \mathrm{~d})$, the scaling used by most previous authors, involving $z_{c}$, is adopted. In Fig. 5(b), the vertical lines correspond to the locations of the drag maxima from Clark and Peltier's (1984) and Smith's (1985) theories: respectively $N z_{c} / \pi U_{0}=$ $0.5+n$ and $N z_{c} / \pi U_{0} \approx 1.1+2 n$ (for $N h_{0} / U_{0}=0.5$ ). In Fig. $5(\mathrm{~d})$, the vertical lines result from the $3 \mathrm{D}$ equivalent of Clark and Peltier's theory: $N z_{c} / \pi U_{0} \approx$ $0.25+n\left(N z_{c} / \pi U_{0}=1.25\right.$ corresponds to the first steepening level in flow over an axisymmetric mountain, as observed in Fig. A1 of Miranda and Valente 1997).

Figure 5 shows that, in the nonlinear regime, the dependence of the drag on $R i$ becomes much weaker, although the amplitude of the modulation still increases as $R i$ decreases. This was noted previously (with fewer data to back it) by Bacmeister and Pierrehumbert (1988). However, some of this reduced sensitivity 

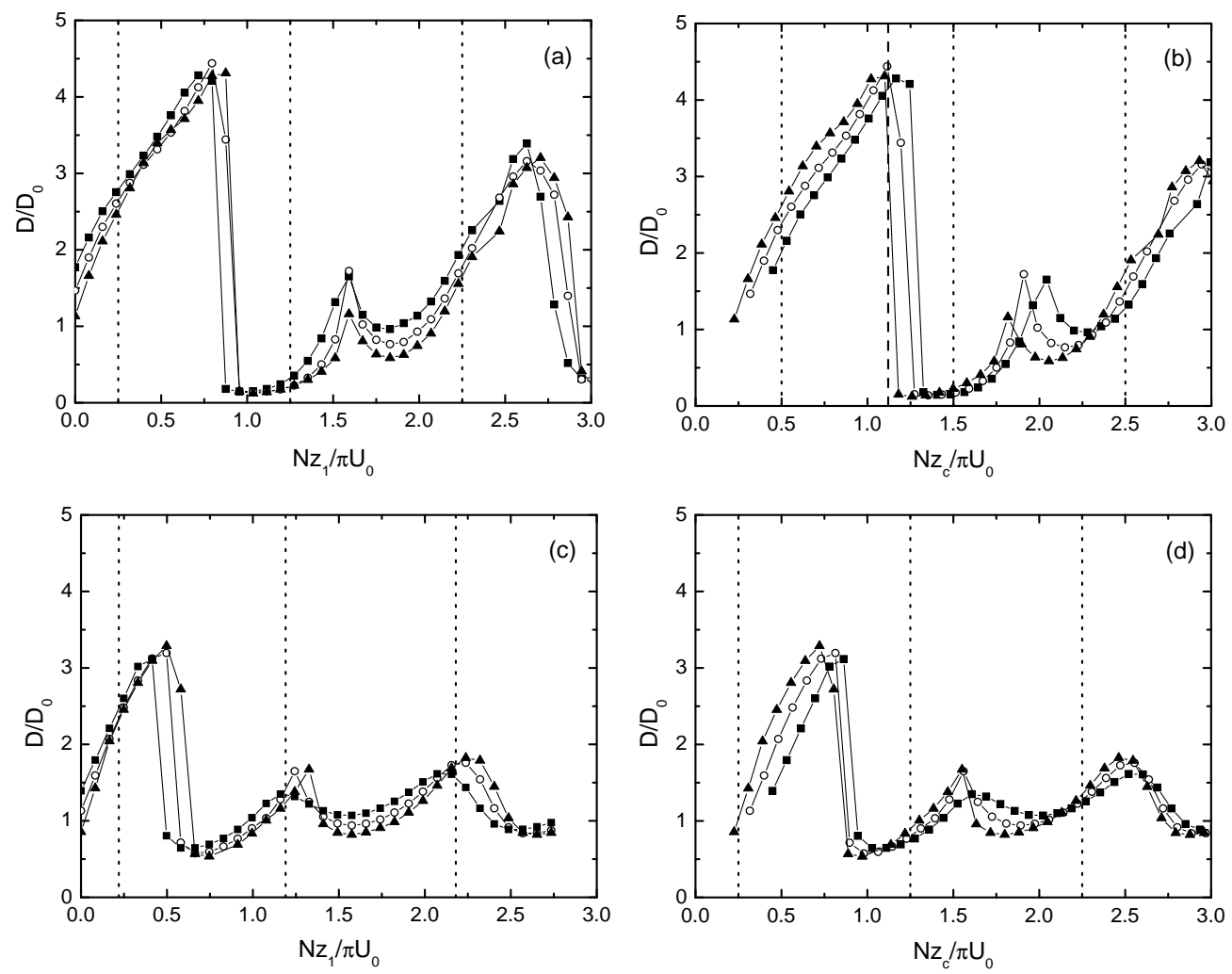

Figure 5. Normalised drag as a function of shear layer height or critical level height for $N h_{0} / U_{0}=0.5$, from numerical simulations. Squares: $R i=2$, circles: $R i=1$, triangles: $R i=0.5$, vertical lines: predicted locations of the maxima. (a) 2D ridge, dotted lines: linear theory, (b) 2D ridge, dotted lines: Clark and Peltier (1984), dashed line: Smith (1985), (c) axisymmtric mountain, dotted lines: linear theory, (d) axisymmetric mountain, dotted lines: analogy with Clark and Peltier (1984).

is relative, since the drag modulation is of considerably larger amplitude than in the linear regime. Indeed, the dependence of the drag on $N z_{1} / \pi U_{0}$ (or $N z_{c} / \pi U_{0}$ ) is stronger, especially in flow over a ridge (see Figs. 5(a,b)) and especially for the first drag maximum. Although in Figs. 5(a,b) the drag curves collapse better using $N z_{1} / \pi U_{0}$ as the horizontal-axis variable, the locations of the maxima are not in agreement with the present model nor with Clark and Peltier's model (cf. Fig. 2 of Bacmeister and Pierrehumbert 1988). For the $N h_{0} / U_{0}$ used here, a smaller second maximum between the first and third maxima still exists. The larger maxima drift slightly to the right as $R i$ decreases when scaled by $z_{1}$ but drift somewhat to the left when scaled by $z_{c}$, which indicates that the key height is somewhere in between $z_{1}$ and $z_{c}$. The first maximum has a very steep forward slope, indicating that the transition from a high to a low drag state happens very suddenly. This appears to be an intrinsically nonlinear effect. This maximum is only predicted adequately by Smith's theory, supporting the idea that the flow is then in a hydraulic regime.

Looking at Figs. 5(c,d), for flow over an axisymmetric mountain, three clearly defined (and comparable in size) drag maxima can be seen, although the second maximum is slightly reduced, especially for $R i=2$. This may be an incipient 

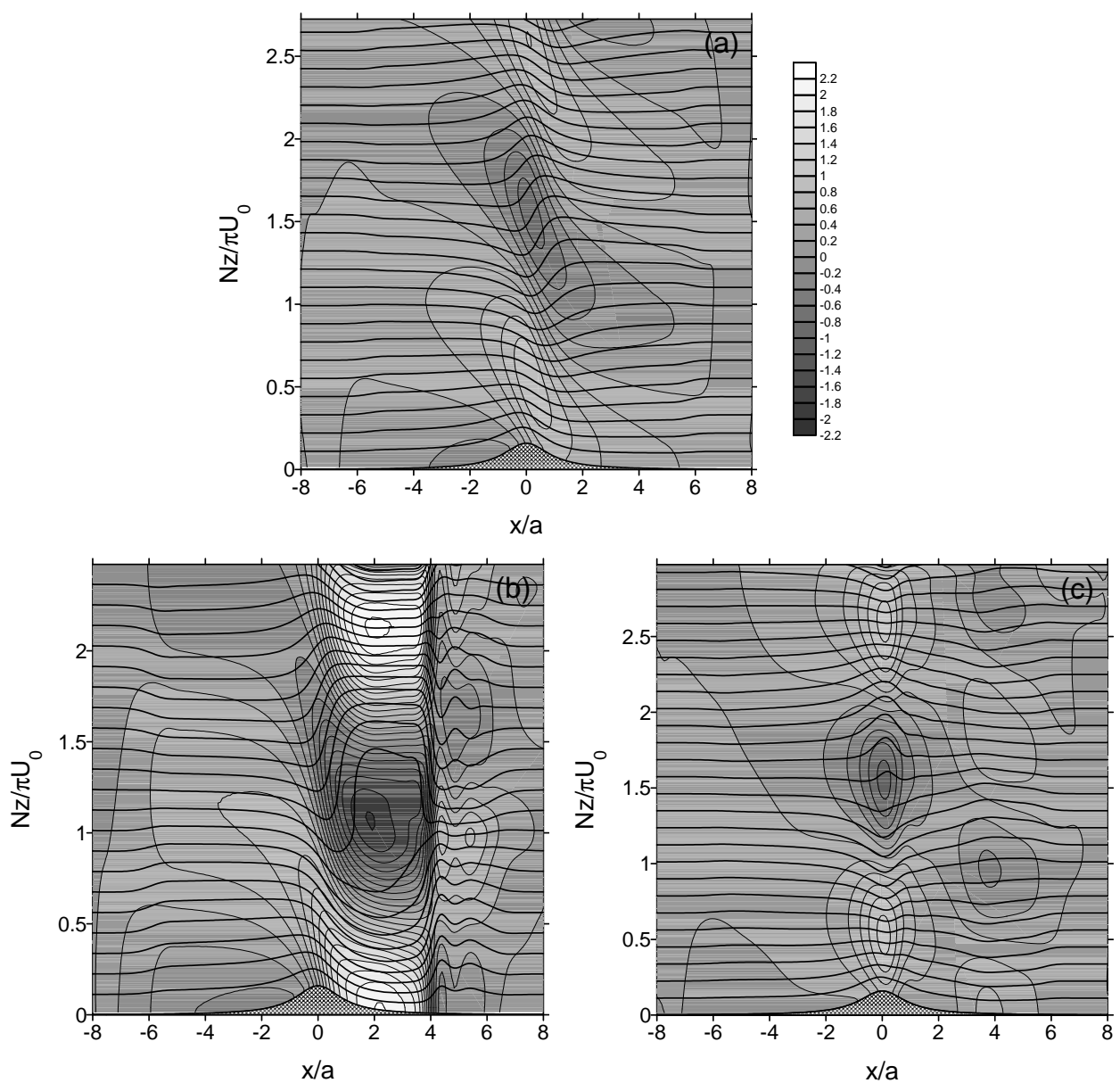

Figure 6. Vertical cross-sections of the normalised streamwise velocity perturbation, $u /\left(N h_{0}\right)$, (shaded contours) and total potential temperature for $N h_{0} / U_{0}=0.5$ (thick solid lines), from numerical simulations. Insentrope spacing: $1 \mathrm{~K}$. (a) $R i=\infty$, (b) $R i=0.5$ and $N z_{1} / \pi U_{0}=2.71$, (c) $R i=0.5$ and $N z_{1} / \pi U_{0}=3.02$. Top of the domain as in Figs. 3(a-c).

manifestation of the same process that suppresses this maximum in flow over a ridge. However, the locations of the maxima (except that of the first one) are in acceptable agreement with linear estimates based on $z_{1}$ (the present model). The first maximum is much larger than the following, and has a steep forward slope, as in the $2 \mathrm{D}$ case. Also here, the maxima drift slightly to the right when scaled by $z_{1}$ and to the left when scaled by $z_{c}$ as $R i$ decreases. The linear prediction for the location of the first drag maximum would be slightly better if the flow were assumed to be lifted by an amount equal to the mountain height, but this procedure seems questionable. Since there is as yet no equivalent of Smith's theory for 3D flow, no further theoretical predictions for the locations of the maxima are available.

Figure 6 shows vertical cross-sections of $u /\left(N h_{0}\right)$ and of the isentropes for flow over a $2 \mathrm{D}$ ridge, for $N h_{0} / U_{0}=0.5$, calculated with the FLEX numerical model. The conditions are similar to those considered in Figs. 5(a,b). By analogy with Fig. 3, a no-resonance case (Fig. 6(a)), an on-resonance case (Fig. 6(b)) and 
an off-resoannce case (Fig. 6(c)) are displayed. Although the on-resonance case corresponds to the third peak in Fig. 5(a,b), and the off-resonance case to the following trough, the location of these features in terms of $N z_{1} / \pi U_{0}$ is not the same as in Fig. 3 due to nonlinear effects.

In Fig. 6(a) it may be seen that the wave structure in the case of no resonance is quite similar to the structure of the linear wave. But in Fig. 6(b) it is apparent that high-drag states are strongly modified by nonlinear effects, with only the downstream lobes of the $u$ minima and maxima intensifying, while the upstream lobes weaken. Since the minima coincide with stagnation zones, this might explain why the second drag maximum tends to disappear in Fig. 5(b). Fig. 6(c) shows that, in the nonlinear low-drag state, the wave propagation is even more suppressed than in linear conditions, but the differences to Fig. 3 are smaller.

The previous results seem to confirm the claim by Wang and Lin (1999b) that linear resonance initiates the high-drag states and that nonlinear processes then modify these states. Although Wang and Lin have advanced some ideas as to how this transition occurs, there is still much to be understood about this phenomenon. Preliminary results (not shown) suggest that, as $N h_{0} / U_{0}$ increases, the amplitude of the drag modulation first increases, and only then do the extrema start to shift their location.

So, how can linear theory help to explain the qualitative differences existing between 3D and 2D flow? Since the drag modulation has a larger amplitude in the $2 \mathrm{D}$ case due to the absence of wave dispersion, the associated flow perturbations also have larger amplitude, being forced outside the linear regime in the 2D case for all the drag maxima and in the 3D case for the first maximum. On the other hand, since the second and third drag maxima in the 3D case are smaller due to wave dispersion, the associated flow perturbations are weaker and the flow remains within the linear regime. Linear theory would also suggest that the flow becomes more nonlinear as $R i$ decreases, because the amplitude of the drag modulation is predicted to increase in that case. This would seem to be consistent with Fig. 2 of Wang and Lin (1999b), where it is noted that the threshold value of $N h_{0} / U_{0}$ for high-drag states is lowered as $R i$ decreases. However, because the dependence of the drag on $R i$ is much reduced in nonlinear conditions, this effect is probably weak, and is not clearly confirmed by Fig. 5 .

\section{Conclusions}

In this study, high-drag states have been investigated in stratified flow over an axisymmetric mountain and a 2D ridge. Using a linear, hydrostatic, analytical model, it was shown that, for a wind profile that is constant near the ground and then decreases linearly with height, high-drag states exist even for infinitesimal dimensionless mountain heights (a possibility considered, but never proved, by Scinocca and Peltier 1991 and Bacmeister and Pierrehumbert 1988). These are only high-drag states in a relative sense since the absolute value of the drag is of course very small (due to the infinitesimal amplitude of the mountains). However, they give some clues about how high-drag states arise in mountains of larger amplitude. The model shows that, in the linear regime, the drag normalised by its value for a constant wind only depends on two dimensionless parameters of the flow: the dimensionless height of the discontinuity in the first derivative of the wind velocity, $N z_{1} / \pi U_{0}$, and the Richardson number in the shear layer 
above, $R i$. The drag oscillates with the first parameter, having a period (when expressed in terms of $z_{1}$ ) of half the hydrostatic vertical wavelength, $\pi U_{0} / N$. The amplitude of this modulation increases as $R i$ decreases. For flow over a $2 \mathrm{D}$ ridge, the drag maxima are located at $N z_{1} / \pi U_{0}=0.25+n$ (where $n$ is an integer), and their magnitude becomes infinite as $R i \rightarrow 1 / 4$. For flow over an axisymmetric mountain, the maxima occur at slightly lower values of $N z_{1} / \pi U_{0}$, and decrease in magnitude as $N z_{1} / \pi U_{0}$ increases, attaining finite values for $R i=1 / 4$. This behaviour is due to internal gravity wave reflection at $z_{1}$. Highdrag states are characterised by constructive interference of the upward and downward propagating waves in the constant-wind layer, while low-drag states correspond to destructive interference.

Nonlinear numerical simulations show that, for a mountain with a moderate dimensionless height $\left(N h_{0} / U_{0}=0.5\right)$, the amplitude of the drag modulation is considerably larger than in the linear regime. However, the drag appears to be much less sensitive to the environmental Richardson number. The locations of the maxima are also shifted to higher $N z_{1} / \pi U_{0}$ relative to the linear prediction, with this shift being particularly pronounced in flow over a $2 \mathrm{D}$ ridge. In that case, the location of the first maximum can only be predicted adequately using Smith's (1985) theory, suggesting that the flow is in a hydraulic regime. In flow over an axisymmetric mountain, the purely linear wave regime (with reflection at $z_{1}$ ) is approximately valid and is only inappropriate for the first drag maximum. This approximate linearity of the flow is also consistent with the fact that the second drag maximum does not tend to disappear (as in flow over a $2 \mathrm{D}$ ridge), and is attributed here to the existence of wave dispersion.

These results suggest that the important height determining the drag maxima changes from $z_{1}$ (predicted by the present linear model) to $z_{c}$ (predicted by Smith's hydraulic model) as $N h_{0} / U_{0}$ increases. It would be interesting to understand how this transition occurs, and what are the underlying physical processes. That undertaking is left for future studies.

\section{ACKNOWLEDGEMENTS}

This work was supported by Fundação para a Ciência e Tecnologia (FCT) under project BULET/33980/99, co-financed by the European Union under program FEDER. MACT acknowledges the financial support of FCT under Grant $\mathrm{SFRH} / \mathrm{BPD} / 3533 / 2000$.

Argain, J. L.

Bacmeister, J. T. and Pierrehumbert, R. T. Clark, T. L. and Farley, R. D.

Clark, T. L. and Peltier, W. R.

Durran, D. R.

\section{REFERENCES}

2003

1988

1984

1984

1992
Numerical modelling of atmospheric flow: orographic and boundary layer effects. PhD thesis, University of $\mathrm{Al}-$ garve, Portugal (in Portuguese)

On the high-drag states of nonlinear stratified flow over an obstacle. J. Atmos. Sci., 45, 63-80

Severe downslope windstorm calculations in two and three spatial dimensions using anelastic interactive grid nesting: a possible mechanism for gustiness. J. Atmos. Sci., 41, 329-350

Critical level reflection and the resonant growth of nonlinear mountain waves. J. Atmos. Sci., 41, 3122-3134

wo-layer solutions to Long's equation for vertically propagating mountain waves: how good is linear theory? Quart. J. Roy. Meteor. Soc., 118, 415-433 
Grimshaw, R. H. and Smyth, N. 1986

Grubišić, V. and Smolarkiewicz, 1997 P. K.

Keller, T. L.

1994

Klemp, J. B. and Lilly, D. K.

Leutbecher, M.

Lindzen, R. S. and Tung, K.-K.

Miles, J. W.

Miranda, P. M. A.

Miranda, P. M. A. and James, I. 1992 N.

Miranda, P. M. A. and Valente, M. A.

Peltier, W. R. and Clark, T. L. 1979

Peltier, W. R. and Clark, T. L. 1983

Phillips, D. S.

Press, W. H., Flannery, B. P., Teukolsky, S. A. and

Vetterling, W. T.

Queney, P.

Sawyer, J. S.

Scinocca, J. F. and Peltier, W. R.

Smith, R. B.

Smith, R. B.

Smith, R. B.

Teixeira, M. A. C., Miranda, P M. A. and Valente, M. A.

Wang, T.-A. and Lin, Y.-L.

Wang, T.-A. and Lin, Y.-L.

Wurtele, M. G., Sharman, R. D. 1996 and Datta, A.
1948

1962

1991

1980

1985

1986

2004

$1999 a$

$1999 \mathrm{~b}$

Resonant flow of a stratified fluid over topography. J. Fluid Mech., 169, 429-464

The effect of critical levels on 3D orographic flows: linear regime. J. Atmos. Sci., 54, 1943-1960

Implications of the hydrostatic assumption on atmospheric gravity waves. J. Atmos. Sci., 51, 1915-1929

1975 The dynamics of wave-induced downslope windstorms. J. Atmos. Sci., 32, 320-339

2001 Surface pressure drag for hydrostatic two-layer flow over axisymmetric mountains. J. Atmos. Sci., 58, 797-807

1976 Banded convective activity and ducted gravity waves. Mon. Wea. Rev., 104, 1602-1617

1961 On the stability of heterogeneous shear flows. J. Fluid Mech., 10, 496-508

Gravity waves and wave drag in flow past three-dimensional isolated mountains. PhD Thesis, University of Reading, $190 \mathrm{pp}$

Non-linear three dimensional effects on the wave drag: splitting flow and breaking waves. Quart. J. Roy. Meteor. Soc., 118, 1057-1081

Critical level resonance in three-dimensional flow past isolated mountains. J. Atmos. Sci., 54, 1574-1588

The evolution and stability of finite-amplitude mountain waves. Part II: surface wave drag and severe downslope windstorms. J. Atmos. Sci., 36, 1498-1529

Nonlinear mountain waves in two and three spatial dimensions. Quart. J. Roy. Metoeor. Soc., 109, 527-548

1984 Analytical surface pressure and drag for linear hydrostatic flow over three-dimensional elliptical mountains. $J$. Atmos. Sci., 41, 1073-1084

1992 Numerical Recipes in Fortran, Cambridge University Press

The problem of airflow over mountains: A summary of theoretical studies. Bull. Am. Meteorol. Soc., 29, 1626

Gravity waves in the atmosphere as a 3-dimensional problem. Quart. J. Roy. Meteor. Soc., 88, 412-425

On the Richardson number dependence of nonlinear criticallayer flow over localized topography. J. Atmos. Sci., 48, $1560-1572$

Linear theory of stratified hydrostatic flow past an isolated obstacle. Tellus, 32, 348-364

On severe downslope windstorms. J. Atmos. Sci., 42, 25972603

Further development of a theory of lee cyclogenesis. J. Atmos. Sci., 43, 1582-1602

An analytical model of mountain wave drag for wind profiles with shear and curvature. J. Atmos. Sci., 61, 1040-1054

Wave ducting in a stratified shear flow over a twodimensional mountain. Part I: general linear criteria. $J$. Atmos. Sci., 56, 412-436

Wave ducting in a stratified shear flow over a twodimensional mountain. Part II: Implications for the development of high-drag states for severe downslope windstorms. J. Atmos. Sci., 56, 437-452

Atmospheric lee waves. Ann. Rev. Fluid Mech., 28, 429-476 